controlled conditions gives a good indication of the fluorine content of coal and of the distribution of this element over the coal components.

It has been found that the fluorine content of natural coal dust (containing most of the fusain) is much higher than that of the dust-free coal (vitrain, clarain, durain). This observation indicates that the fluorine is derived from the water which, according to accepted theories, has furnished by a process of infiltration the bulk of the mineral constituents of fusain. On putting this theory to the test, it was found that a small portion of fluorine could be extracted by water and a larger portion by a 1 per cent solution of sodium hydroxide. This distinction proves that the fluorine is mainly present in the form of calcium fluoride.

In one of the coals examined, the chloride content in the dust was three to four times higher than in the dust-free clean coal. As a similar ratio appears to exist between the fluorine contents of the two materials, it is fairly certain that the water with which the coal substance was in contact during or after its formation must be regarded as the source of this element in coal. Moreover, the ratio of fluorine to chlorine in one of the coals examined is of the same order as that found in sea-water. The discovery of fluorine in coals will therefore prove of interest in the study of coal formation.

Its practical importance lies in the direction of eliminating coal components, notably dust, from processes in which an accumulation of fluoride might cause difficulties, as in the case which gave rise to this investigation.

R. LESSING.

50 Queen Anne's Gate, London, S.W.1.

\section{Conception of 'Synthesis' in Organic Chemistry}

Ir is perhaps ungrateful to take exception even in part to so appreciative a note as that on "The Male Sex Hormone" which appeared in NATuRE of October 13 (p. 563). However, it contains the following sentence: "It is unfortunate that this conversion of cholesterol into androsterone should be described as a "synthesis'." Now, the elimination of water from ethyl alcohol is designated as a synthesis of ethylene, and the pyrogenetic decomposition of dipentene is a synthesis of isoprene. These 'partial syntheses' become 'complete' when the starting materials, ethyl alcohol, dipentene, and, in the case of the male sex hormone, epidihydrocholesterol, can be built up from the elements. Naturally, the term 'synthesis' should not be too freely used, but we are nevertheless of the opinion that the first artificial preparation of a sex hormone from a compound with a different number of carbon atoms merits this designation, particularly as we clearly stated in the title of our paper: "Synthese ... durch Abbau. ..." L. RuzickA.

Laboratory for Organic Chemistry, Technical High School, Zurich.

My opinion is in no way altered by Prof. Ruzicka's comments. When Prof. Ruzicka has succeeded in preparing epidihydrocholesterol from carbon, hydrogen and oxygen (or from some compound which can be shown to be capable of artificial formation from these elements), I shall endeavour to be the first to congratulate him on having synthesised the male sex hormone (androsterone). I am not concerned with the question whether the transformation of the sterol into the hormone involves a 'building up' or a 'breaking down' of the molecule. The main issue, in my view, is that the term 'synthesis' cannot be justified, either by definition (see, for example, Bailey and Bailey : "An Etymological Dictionary of Chemistry and Mineralogy", 1929) or by common usage, as a description of the conversion into another substance of a compound of purely natural origin. I am sure that the great majority of chemists will agree with this point of view.

In saying this, I am not disparaging Prof. Ruzicka's magrificent achievement. I still have very pleasant memories of a few brief hours spent in his company last March, when he was kind enough to tell me something of the early experiments which have been brought to such a successful conclusion.

The Writer of the Note.

The Orthogonal Matrix transforming Spearman's Two-Factor Equations into Thomson's Sampling Equations in the Theory of Ability

IF $\varphi$ is a column vector of $t$ elements representing scores in $t$ tests, and $s$ a column vector of $t+1$ elements of which the first, $s_{0}$, represents Spearman's $g$ and the rest his specific factors, then $\varphi=L_{s}$ represents $t$ Spearman equations giving the composition of the $\varphi^{\prime} s$, which will in that case be perfectly hierarchical. Here $L$ is an oblong matrix of $t$ rows and $t+1$ columns. Its first column is $\left\{l_{1} l_{2} \ldots l_{t}\right\}$ where $l_{i}$ is the correlation of $\varphi_{i}$ with $g$. The principal diagonal of the remainder of $L$ is $\left(m_{1} m_{2} \ldots m_{t}\right)$ where $m_{i}^{2}=1-l^{2} i$. The remaining elements in $L$ are zero.

The transformation $s=\bar{O} y$, where $\bar{O}$ is a slab of an orthogonal matrix $O$, will transform these Spearman equations into equivalent equations $\varphi=L \vec{O} y$ (where the components $y$ may be of any number not less than $t$ ) which will give the same correlations as before. Among the infinity of matrices $O$ there is one, $T$, the proper slab of which transforms the Spearman equations into equations agreeing with the most probable result of the Sampling Theory, on which theory the complete set of tests formed from all possible linear combinations of the components will be strongly though not perfectly hierarchical, so that a perfectly hierarchical sample can easily be selected, as is in fact done in practice.

The matrix $T$ will be described more fully elsewhere. It is of order $2^{t}$ (the slab required being the first $t+1$ rows), is composed of elements like $\pm l_{1} l_{2} m_{3} l_{4} \ldots m_{t}$, is axisymmetrical, and has a special kind of reflex symmetry about its vertical and horizontal centre-lines, which can be most readily followed when it is divided into "binomial" com. partments by taking the $2 t$ rows (and columns) in blocks of $1, t, t ! /(t-2) ! 2 ! \ldots$ (the binomial coefficients). I arrived at it by other means, but Dr. A. C. Aitken has since pointed out to me that it is a Zehfuss matrix and can be made by multiplicative composition (called in America the direct product) of $t$ matrices each of the form

$$
\left[\begin{array}{cr}
l_{i} & m_{i} \\
m_{i} & -l_{i}
\end{array}\right]
$$

University,

Edinburgh.

Godfrey H. Thomson.

Oct. 6 . 